\title{
Image Retrieval using Quantized Local Binary Pattern
}

\author{
P. Latha \\ M.Tech, Anurag \\ Group of Institutions \\ (Autonomous), \\ Hyderabad, Telangana, India
}

\author{
V. Vijaya Kumar \\ Professor, Dean and \\ Director for CACR, \\ Anurag Group of \\ Institutions (Autonomous), \\ Hyderabad, Telangana, India
}

\author{
A. Obulesu \\ Asst.Professor, \\ Anurag Group of \\ Institutions (Autonomous), \\ Hyderabad, Telangana, India
}

\begin{abstract}
Image retrieval is one of the main topics in the field of computer vision and pattern recognition. Local descriptors are gaining more and more recognition in recent years as these descriptors are capable enough to identify the unique features, which suitably and uniquely describe any image for recognition and retrieval. One of the popular and efficient frame works for capturing texture information precisely is the Local binary pattern (LBP). LBP descriptors perform well in varying pose, illumination and lighting conditions. LBP is a structural approach and plays significant role in wide range of applications. One of the disadvantages with LBP based frame work is its dimensionality. The dimensionality of LBP increases, if one increases the number of neighboring pixels. Further statistical approaches gained lot of significance in image retrieval and LBP based methods raises high dimensionality and complexity issues, in deriving statistical features. The present paper addresses these two issues by quantizing the LBP code, to reduce dimensionality and by deriving GLCM features on quantized LBP. The proposed method is experimented on Corel database and compared with other existing methods. The experimental results indicate the high retrieval rate by the proposed method over the existing methods.
\end{abstract}

\section{Keywords}

Structural; Statistical approach; Pose; Illumination; Dimensionality;

\section{INTRODUCTION}

The need for an efficient system to facilitate users in searching and organizing image collections in a large scale database is crucial. However, developing such systems is quite difficult, because an image is an ill-defined entity consisting of complex and highly variable structures. Hence there is a need of some automatic search technique called content based image retrieval (CBIR). The feature extraction is a very important step and the effectiveness of a CBIR system depends typically on the method of features extraction from raw images. The CBIR uses the visual contents of an image such as color, texture, shape, faces, spatial layout etc. to represent and index the image. The visual features can be further classified as general features (color, texture and shape) and domain specific features as human faces and finger prints. There is no single best representation of an image for all perceptual subjectivity, because the user may take the photographs in different conditions (view angle, illumination changes etc.). Comprehensive and extensive literature survey on CBIR is presented in [1]-[4]. CBIR aims at developing techniques that support effective searching and browsing of large image digital libraries on the basis of automatically derived image features [5, 6]. Swain et al. [7] proposed the concept of color histogram in 1991 and also introduced the histogram intersection distance metric to measure the distance between the histograms of images. Stricker et al. [8] used the first three central moments called mean, standard deviation and skewness of each color for image retrieval. Pass et al. [9] introduced color coherence vector $(\mathrm{CCV}) . \mathrm{CCV}$ partitions the each histogram bin into two types, i.e., coherent, if it belongs to a large uniformly colored region or incoherent, if it does not. Huang et al. [10] used a new color feature called color correlogram which characterizes not only the color distributions of pixels, but also spatial correlation of pair of colors. Lu et al.[11] proposed color feature based on vector quantized (VQ) index histograms in the discrete cosine transform (DCT) domain. They computed 12 histograms, four for each color component from 12 DCT-VQ index sequences. Furthermore, there is no single universal approach to the content-based retrieval problems, even in a restricted domain application [5]. There are systems that discuss about the objects based on similarity measures. Based on the similarity of each object set in each group (also called a cluster) or they are not similar are put into different clusters. CLUE, cluster based retrieval of images by unsupervised learning is an example of CBIR technique based on unsupervised learning $[12,13,14]$. Texture plays an important role in image classification, retrieval, segmentation and age classification. In Literature various texture based methods are derived for image classification [ $15,16,17]$, face recognition $[18,19,20]$, age and facial expressions identification [21, 22], segmentation [23, 24, 25] image retrieval[26, 27, 28, 29, 30, 31,32 ]. Texture is salient and indispensable feature for CBIR. Smith et al. used the mean and variance of the wavelet coefficients as texture features for CBIR [33]. Moghaddam et al. proposed the Gabor wavelet correlogram (GWC) for CBIR $[34,35]$. Ahmadian et al. used the wavelet transform for texture classification [36]. Moghaddam et al. introduced new algorithm called wavelet correlogram (WC) [37]. Saadatmand et al. $[38,39]$ improved the performance of WC algorithm by optimizing the quantization thresholds using genetic algorithm (GA). Birgale et al. [40] and Subrahmanyam et al. [41] combined the color (color histogram) and texture (wavelet transform) features for CBIR. Subrahmanyam et al. proposed correlogram algorithm for image retrieval using wavelets and rotated wavelets (WC+RWC) [42].

The rest of the paper organized as follows: Section 2 gives the proposed algorithm, section 3 describes about results and discussions and finally section 4 conclude the paper.

\section{METHODOLOGY}

The present paper intends to reduce the dimensionality and complexity issues of LBP coded image while preserving the significant local texture features precisely and accurately. The present paper after an in-depth survey on the CBIR methods, found that there is a necessity to integrate second order statistical features with structural features to design an efficient and precise image retrieval model. To implement this the present paper derived a new approach for CBIR and is given below and it consist of seven steps 
Step One: Compute color histograms of the images using RGB vectors.

Step Two: Convert the color image in to gray scale image using RGB color space. The conversion mechanism using RGB color space model is described below.

In order to extract gray level features from color information, the proposed method utilized the RGB color space which quantizes the color space into 7-bins to obtain 128 gray levels. The index matrix of 128 color image is denoted as $\mathrm{C}(\mathrm{x}, \mathrm{y})$. The RGB quantization process is done by using 7-bit binary code of 128 colors as given in Eqn. (1) to (4).

$$
\begin{gathered}
\mathrm{C}(\mathrm{x}, \mathrm{y})=16 * \mathrm{I}(\mathrm{R})+2 * \mathrm{I}(\mathrm{G})+\mathrm{I}(\mathrm{B}) \\
\text { where } \\
\mathrm{I}(\mathrm{R})=0,0 \leq \mathrm{R} \leq 16, \mathrm{I}(\mathrm{R})=\mathrm{i},\left(\left(16^{*} \mathrm{i}\right)+1\right) \leq \mathrm{R} \leq\left(16^{*}(\mathrm{i}+1)\right) \\
\mathrm{i}=[1,2, \ldots, 7] \\
\mathrm{I}(\mathrm{G})=0,0 \leq \mathrm{G} \leq 16, \mathrm{I}(\mathrm{G})=\mathrm{i},((16 * \mathrm{i})+1) \leq \mathrm{G} \leq\left(16^{*}(\mathrm{i}+1)\right. \\
\mathrm{i}=[1,2, \ldots, 6] \\
\mathrm{I}(\mathrm{B})=0,0 \leq \mathrm{B} \leq 32, \mathrm{I}(\mathrm{B})=\mathrm{i},((32 * \mathrm{i})+1) \leq \mathrm{B} \leq(32 *(\mathrm{i}+1) \\
\mathrm{i}=[1,2,3]
\end{gathered}
$$

Therefore, each value of $\mathrm{C}(\mathrm{x}, \mathrm{y})$ is a 7 bit binary code ranging from 0 to 127 .

Step Three: Derivation of the LBP coded image: This is accomplished by computing the LBP code on each $3 \times 3$ neighborhood in an overlapped manner and by replacing the central pixel with LBP coded image. The LBP code ranges from 0 to 255. If one constructs GLCM on this LBP coded image then the size of the matrix will be $256 \times 256$ ( 0 to $255 \times$ 0 to 255 )

The LBP operator scans the image in an overlapped manner based on a $3 \times 3$ neighborhood. The LBP initially or in first step converts the neighboring pixels of $3 \times 3$ neighborhood in to a binary code based on a threshold. The threshold is mostly the gray level value of the central pixel. In the second step the binary weights are given to the neighboring pixels. The summation of these neighboring pixels with their binary values is multiplied by the corresponding binary weights and this results a weighted value. The summation of all these weighted values of 8-neighboring pixels results a LBP code. In step three the central pixel value is replaced by the LBP code. This process is repeated on entire image in a non-overlapped manner and the image is becomes a LBP coded image

A $3 \times 3$ circular neighborhood consists of a set of nine elements, $\mathrm{K}=\left\{\mathrm{K}_{\mathrm{c}}, \mathrm{K}_{0}, \mathrm{~K}_{1}, \ldots, \mathrm{K}_{7}\right\}$, where $\mathrm{K}_{\mathrm{c}}$ and $\mathrm{K}_{\mathrm{i}}(0 \leq \mathrm{i} \leq 7)$ correspond to the gray values of the central pixel and peripheral pixels. The neighborhood can be denoted by a set of binary values $B_{i}$ $(0 \leq i \leq 7)$ as given in equation.

$$
\begin{gathered}
b_{i}=\left\{\begin{array}{cc}
0 & \Delta p_{i} \geq 0 \\
1 & \Delta p_{i}<0
\end{array}\right. \\
\text { where } \Delta \mathrm{K}_{\mathrm{i}}=\mathrm{K}_{\mathrm{i}}-\mathrm{K}_{\mathrm{c}}
\end{gathered}
$$

For each $3 \times 3$ neighborhood, a unique LBP code is derived from the equation 6

$$
L B P_{P, R}=\sum_{i=0}^{i=7} b_{i} \times 2^{i}
$$

Every pixel in an image generates an LBP code. A single LBP code represents local micro texture information around a pixel by a single integer code LBP $\epsilon[0,255]$.

\begin{tabular}{|c|c|c|c|c|c|}
\hline 141 & 143 & 141 & 0 & 1 & 0 \\
\hline 141 & 142 & 143 & 0 & & 1 \\
\hline 141 & 141 & 141 & 0 & 0 & 0 \\
\hline \multicolumn{3}{|c|}{$\begin{array}{l}\text { Fig.2(a): Actual } \\
\text { LBP image }\end{array}$} & \multicolumn{3}{|c|}{$\begin{array}{c}\text { Fig.2(b) : } \\
\text { Generation of } \\
\text { Binary } \\
\text { atterns Based on } \\
\text { Equation } 5\end{array}$} \\
\hline
\end{tabular}
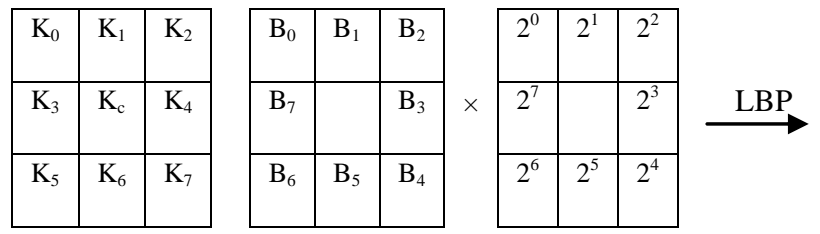

Fig.1: Representation of LBP.

The $\mathrm{LBP}_{\mathrm{P}, \mathrm{R}}$ constructs $2^{\mathrm{P}}$ dissimilar output values, related to the $2^{\mathrm{P}}$ different binary patterns that can be created by the $\mathrm{P}$ surrounding pixels and these are rotation dependent. The derivation of LBP code from a local neighborhood is given below.

The LBP code evaluation process as follows

\begin{tabular}{|l|l|l|}
\hline $2^{0}$ & $2^{1}$ & $2^{2}$ \\
\hline $2^{7}$ & & $2^{3}$ \\
\hline $2^{6}$ & $2^{5}$ & $2^{4}$ \\
\hline
\end{tabular}$\rightarrow 10$

Fig.2(c):Correspondi ng Weights

Fig.2(d): LBP Code

Fig. 2: Derivation of LBP code from a local neighborhood.

LBP is a local texture operator with low computational complexity and low sensitivity to changes in illumination and LBP has the following advantages.

- The local texture character can be described efficiently.

- It is easy to operate and understand.

- The whole image character description can be easily extended.

Step Four: To overcome the high dimensionality problem the present paper quantized the LBP coded image in to 10 levels ranging from 0 to 9 . This reduced the dimension of the GLCM into $10 \times 10$. The quantization process is done using the following equation 7 and by this, the image becomes quantized- LBP (Q-LBP) 


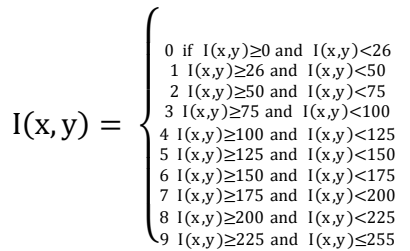

Step Five: Construction of GLCM on quantized LBP coded image. The GLCM is derived with varying distances $d=1,2,3$ and 4. And on each d four GLCMS are constructed with $0^{\circ}$, $45^{\circ}, 90^{\circ}$ and $135^{\circ}$. Thus the present paper derived sixteen GLCM's and four GLCM's on each $d_{i}$ where $i=1,2,3$ and 4 .

The Gray level co-occurrence matrix (GLCM), a second order statistical method, was introduced by Haralick et al. [43] and able to characterize textures based on the spatial relationship between gray tones in an image [44]. Haralick [43] proposed 13 kinds of textural features each extracted from the gray level co-occurrence matrix. In general, GLCM could be computed as follows. Then, GLCM is computed from G by scanning the intensity of each pixel and its neighbor, defined by displacement $d$ and angle $\varnothing$. A displacement, $d$ could take a value of $1,2,3 \ldots n$ whereas an angle, $\varnothing$ is limited $0^{\circ}, 45^{\circ}, 90^{\circ}$ and $135^{\circ}$. In a given distance $\mathrm{d}$ and direction given by, the Gray Level Co-occurrence matrix is calculated by using gray scale pixel $\mathrm{i}$ and $\mathrm{j}$, expressed as the number of co-occurrence matrix in different directions.

$$
P(i, j \mid d, \theta)=p(i, j \mid d, \theta) \Sigma \Sigma p(i, j \mid d, \theta)
$$

The following Fig. 3 illustrates the formation of GLCM with different angles $\left(0^{\circ}, 45^{\circ}, 90^{\circ}\right.$ and $\left.135^{\circ}\right)$ from a given window.

\begin{tabular}{|l|l|l|}
\hline 3 & 3 & 3 \\
\hline 1 & 3 & 3 \\
\hline 1 & 3 & 2 \\
\hline
\end{tabular}

\begin{tabular}{|c|c|c|c|}
\hline $45^{\circ}$ & 1 & 2 & 3 \\
\hline 1 & 0 & 0 & 2 \\
\hline 2 & 0 & 0 & 0 \\
\hline 3 & 0 & 0 & 2 \\
\hline
\end{tabular}

\begin{tabular}{|c|c|c|c|}
\hline $135^{\circ}$ & 1 & 2 & 3 \\
\hline 1 & 0 & 0 & 0 \\
\hline 2 & 0 & 0 & 1 \\
\hline 3 & 0 & 0 & 2 \\
\hline
\end{tabular}

Fig.3: An example of GLCM formation.

Step Six: Derive GLCM features entropy, energy, contrast, local homogeneity and correlation with $0^{\circ}, 45^{\circ}, 90^{\circ}$ and $135^{\circ}$ on each $d$. The average values of $0^{\circ}, 45^{\circ}, 90^{\circ}$ and $135^{\circ}$ are considered as feature vectors for image retrieval.

The GLCM features are evaluated using following equations:

- Entropy gives measures of complexity of the image and the complex texture tends to higher entropy.

- Energy is the sum of squared elements in the GLCM and it is by default one for constant image.

- Contrast measures intensity between a pixel and its neighbor over the whole image and it is considered zero for constant image and it is also known as variance and moment of inertia.

- Homogeneity is the most commonly used measure that increases with less contrast in the image. If weights decrease away from the diagonal, the result will be larger for windows with little contrast. Homogeneity weights values by the inverse of the Contrast weight, with weights decreasing exponentially away from the diagonal

- Correlation measures how pixel is correlated to its neighbor over the whole image.

$$
\begin{gathered}
\text { Entropy }=\sum_{\mathrm{I}, \mathrm{j}=0}^{\mathrm{N}-1}-\ln \left(\mathrm{P}_{\mathrm{ij}}\right) \mathrm{P}_{\mathrm{ij}} \\
\text { Energy }=\sum_{\mathrm{I}, \mathrm{j}=0}^{\mathrm{N}-1}-\ln \left(\mathrm{I}\left(\mathrm{P}_{\mathrm{ij}}\right)^{2}\right. \\
\text { Contrast }=\sum_{\mathrm{I}, \mathrm{j}=0}^{\mathrm{N}-1} \mathrm{I} \mathrm{P}_{\mathrm{ij}}(\mathrm{i}-\mathrm{j})^{2} \\
\text { Local Homogenity }=\sum_{\mathrm{I}, \mathrm{j}=0}^{\mathrm{N}-1} \frac{\mathrm{P}_{\mathrm{ij}}}{1+(\mathrm{i}-\mathrm{j})^{2}} \\
\text { Correlation }=\sum_{\mathrm{I}, \mathrm{j}=0}^{\mathrm{N}-1} \mathrm{P}_{\mathrm{ij}} \frac{(\mathrm{i}-\mu)(\mathrm{j}-\mu)}{\sigma^{2}}
\end{gathered}
$$

where $P_{i j}$ is the pixel value in position $(I, j)$ of the texture image, $\mathrm{N}$ is the number of gray levels in the image, $\mu$ is $\mu=$ $\sum_{i, j=0}^{N-1} i P_{i j}$ mean of the texture image and $\sigma^{2}$ is $\sigma^{2}=$ $\sum_{i, j=0}^{N-1} P_{i j}(i-\mu)^{2}$ variance of the texture image.

Step Seven: Use similarity distance measure based on color histogram and Q-LBP-GLCM features for comparing the query image feature vector and feature vectors of the database images.

The present retrieval model selects 16 top images from the database images that are matching with query image. And also experimented with more number of top images and retrieval performance is measured. This is accomplished by measuring the distance between the query image and database images. The present paper used Euclidean distance as the distance measure and as given below

$$
\operatorname{Dist}_{s}\left(T_{n}, I_{n}\right)=\left(\sum_{i, j=1}\left|f_{i}\left(T_{n}\right)-f_{j}\left(I_{n}\right)\right|^{2}\right)^{1 / 2}
$$

Where $T_{n}$ query image, $I_{n}$ image in database;

\section{RESULTS AND DISCUSSIONS}

The present paper carried out image retrieval on Corel database [45]. This database consists of a large number of images of various contents ranging from animals to outdoor sports to natural images. These images have been pre classified into different categories each of size 100 by domain professionals. Corel database meets all the requirements to evaluate an image retrieval system, due to its latge size and heterogeneous content. For our experiment, we have collected 1000 images form database compromising 10 classes. That is each class consists of 100 images. The classes of image are displayed in Fig. 4 i.e. African, Sea shore,Tombs, Bus, Dinosaur, Elephants, Fancy Flowers, Horses, Valleys and Evening Skies. Each category has images with resolution of either $256 \times 384$ or $384 X 256$. The performance of the present model is evaluated in terms of precision, recall rate and accuracy as given in equation 15,16 and 17 respectively. Precision is the ratio of number of retrieved images Vs. the number of relevant images retrieved. The recall is the ratio of number of relevant image retrieval Vs. total number of relevant images in the database.

$$
\begin{gathered}
\text { precision }=\frac{\text { Number of relevant images retried }}{\text { Total number of images retrieved }} \\
\text { Recall }=\frac{\text { Number of relevant images retrieved }}{\text { Total number of relevant images in database }} \\
\text { Accuracy }=\frac{\text { precision }+ \text { recall }}{2}
\end{gathered}
$$

The present paper compute GLCM features on Q-LBP using various distance values: $D=1,2,3,4$ and query matching is 
performed using Euclidean distance. The present retrieval model selects 16 top images from the database images that are matching with query image. And also experimented with more number of top images and retrieval performance is measured. Fig. 5(a) - 5(e) shows five examples of retrieval images, i.e. one image from each class, by the proposed method with $\mathrm{D}=4$ for 16 top matched images and top left most image is the query image.

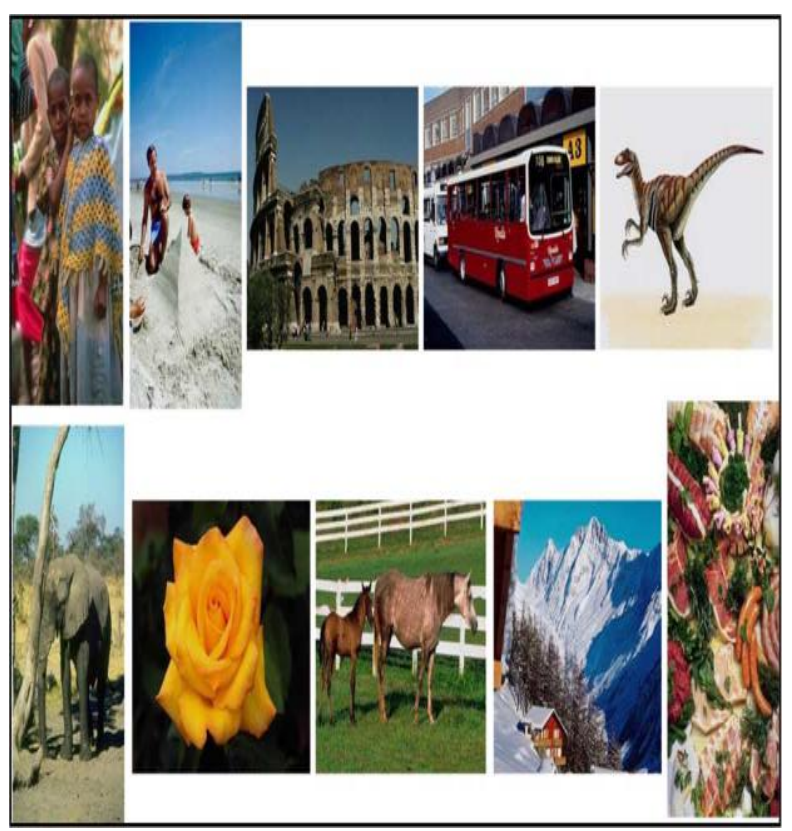

Fig.4: Considered 10 classes of Corel database from top left to bottom right African, Sea shore, Tombs, Bus, Dinosaur, Elephants, Fancy Flowers, Horses, Valleys and EveningSkies.

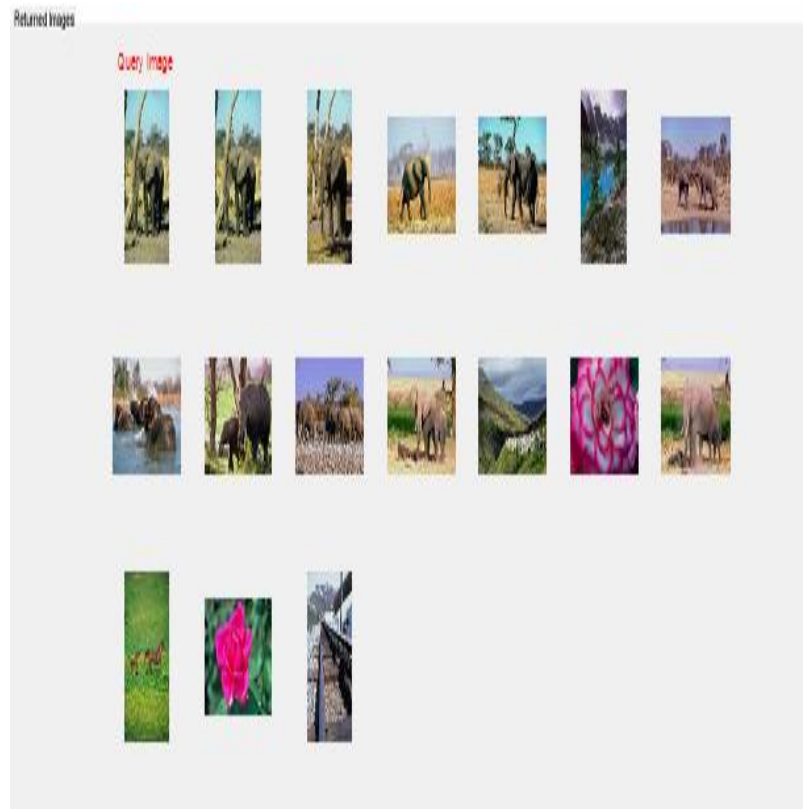

Fig.5(a): Retrieved elephant images by the proposed method.

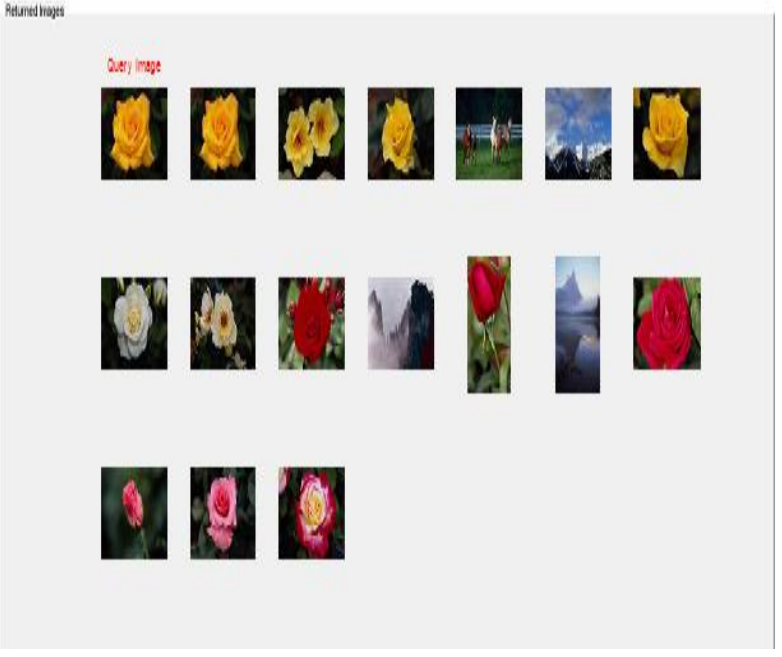

Fig.5(b): Retrieved flower images by the proposed method.

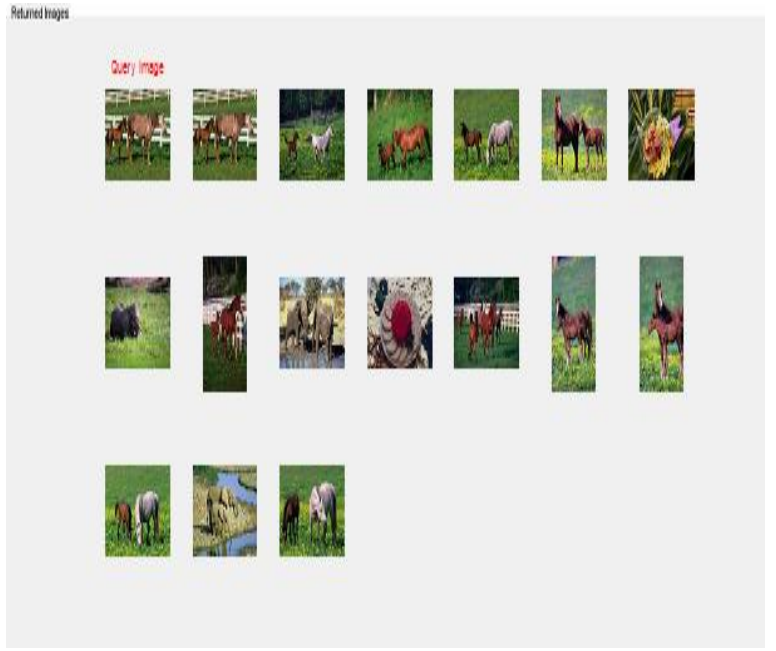

Fig.5(c): Retrieved horse images by the proposed method.

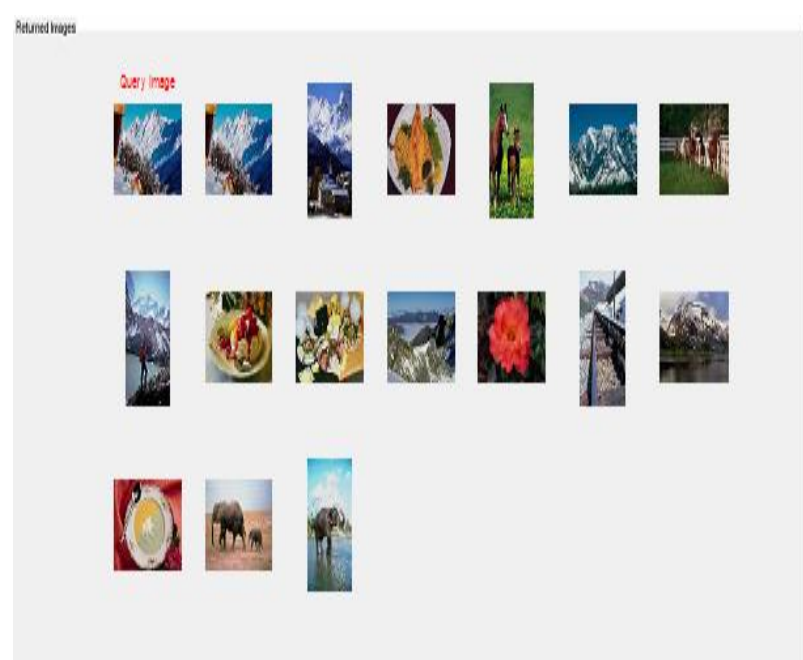

Fig.5(d): Retrieved mountain images by the proposed method. 


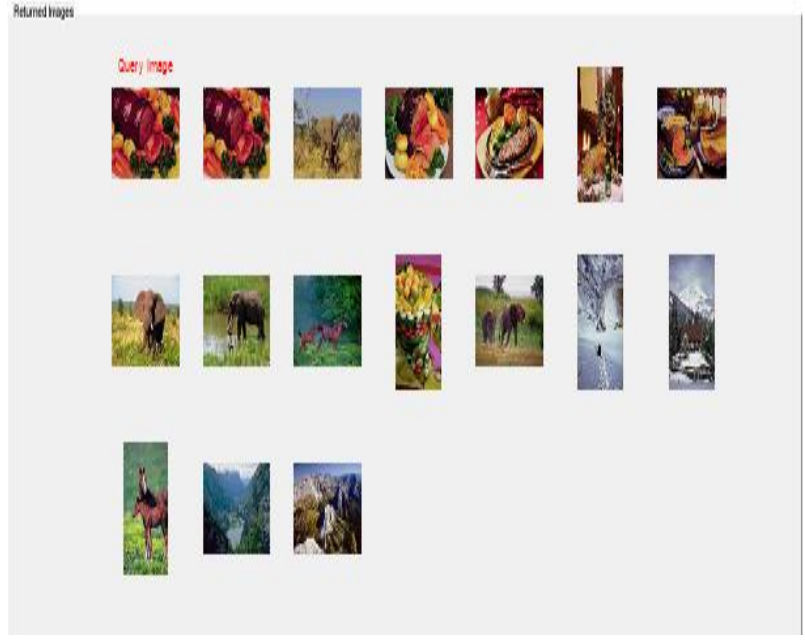

Fig.5(e): Retrieved food item images by the proposed method.

Fig 5(a) to 5(e) Retrieved images for each class with $D=4$ for 16 top matched images on proposed integrated method.

The average precision, recall rates and accuracy computed based on "Q-LBP-GLCM features and color histograms" (integrated Q-LBP) and listed in Tables 1. The best performance of integrated Q-LBP is obtained when $D=4$. The retrieval performance of the integrated Q-LBP is compared with HCA [46], CBIR-C [47] and FCMC [48] methods. The present paper selected 60 images of the same category or class as query images (one by one) and computed precession and recall rates by selecting top $16,20,30,40,50,60,70,80$ and 90 images. The average precession rates of HCA, CBIR-C and FCMC methods are ranging from $38 \%$ to $45 \%, 39 \%$ to $46 \%$ and $60 \%$ to $64 \%$ respectively and for number of images retrieved is 16 (Table 2).

The average precession, recall rates and accuracy of proposed method for different $\mathrm{d}$ values are plotted in graphs, specified in Fig.6, Fig. 7 and Fig.8 respectively.

Table 1: Average precision and recall rate of all classes of images with various distance measures for 16 top matched images

\begin{tabular}{|c|c|c|c|c|c|}
\hline Methods & & \multicolumn{4}{|c|}{ Distance parameter } \\
\cline { 3 - 6 } & & $\mathrm{d}=1$ & $\mathrm{~d}=2$ & $\mathrm{~d}=3$ & $\mathrm{~d}=4$ \\
\hline \multirow{3}{*}{$\begin{array}{c}\text { Integrated } \\
\text { Q-LBP }\end{array}$} & Precision & 0.65 & 0.66 & 0.68 & 0.70 \\
\cline { 2 - 6 } & Recall & 0.34 & 0.36 & 0.41 & 0.46 \\
\cline { 2 - 6 } & Accuracy & 0.50 & 0.51 & 0.55 & 0.59 \\
\hline
\end{tabular}

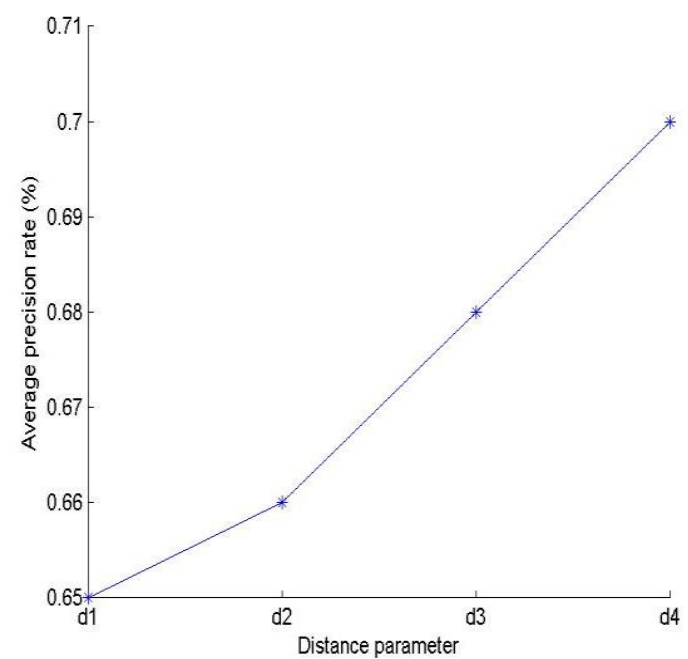

Fig.6: Average precision graph for proposed integrated QLBP for different d values.

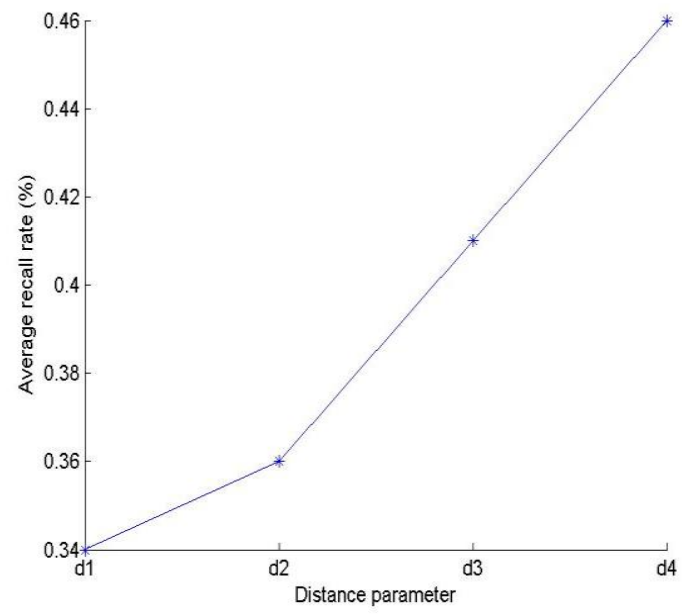

Fig.7: Average recall graph for proposed integrated Q-LBP for different d values.

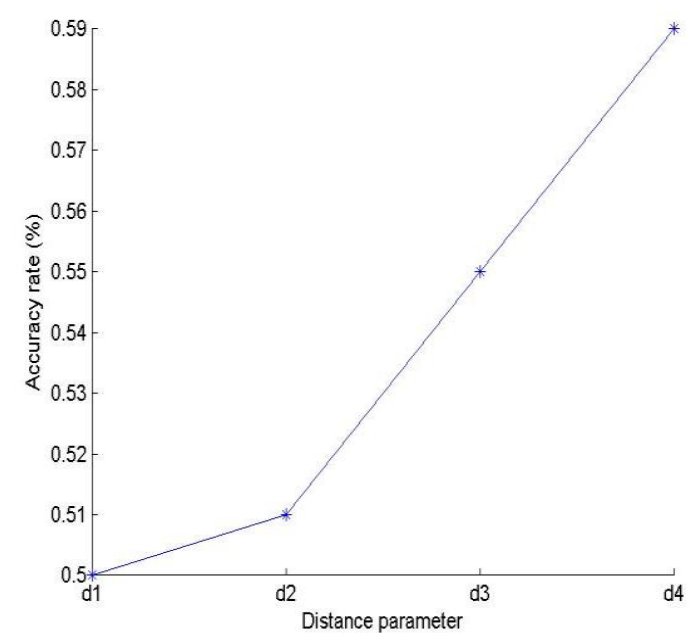

Fig.8: Accuracy graph for proposed integrated Q-LBP for different d values. 
The average precision rate of proposed method QLBP and existing methods i.e. hierarchical clustering algorithms (HCA), Content Based Image Retrieval Using Clustering (CBIR-C), Fuzzy C-Means clustering scheme (FCMC) is given in Table 2 and plotted graphs as shown in Fig. 9, Fig. 10 and Fig.11. From these graphs, it is clearly seen that the proposed integrated QLBP outperforms the HCA, CBIR-C, and FCMC over the considered database using both ARP, ARR and average accuracy evaluation metrics.

Table 2: Average precession rate on each class of images between existing and proposed method for 16 top retrieved images.

\begin{tabular}{|l|c|c|c|c|c|c|}
\hline & \multicolumn{5}{|c|}{ Image category and the precision (\%) } \\
\hline Methods & $\begin{array}{c}\text { Eleph } \\
\text { ants }\end{array}$ & $\begin{array}{c}\text { Fanc } \\
\text { ylo } \\
\text { Fers }\end{array}$ & $\begin{array}{c}\text { Horse } \\
\text { s }\end{array}$ & $\begin{array}{c}\text { Valle } \\
\text { ys }\end{array}$ & $\begin{array}{c}\text { Eveni } \\
\text { ng } \\
\text { Skies }\end{array}$ & $\begin{array}{c}\text { Averag } \\
\text { e }\end{array}$ \\
\hline $\begin{array}{l}\text { HCA } \\
\text { [46] }\end{array}$ & 0.39 & 0.42 & 0.44 & 0.48 & 0.5 & 0.45 \\
\hline $\begin{array}{l}\text { CBIR_C } \\
\text { [47] }\end{array}$ & 0.4 & 0.43 & 0.46 & 0.49 & 0.52 & 0.46 \\
\hline $\begin{array}{l}\text { FCMC[4 } \\
\text { 8] }\end{array}$ & 0.61 & 0.6 & 0.66 & 0.67 & 0.7 & 0.64 \\
\hline $\begin{array}{l}\text { Integrate } \\
\text { d Q- } \\
\text { LBP }\end{array}$ & 0.68 & 0.69 & 0.71 & 0.72 & 0.71 & 0.70 \\
\hline
\end{tabular}

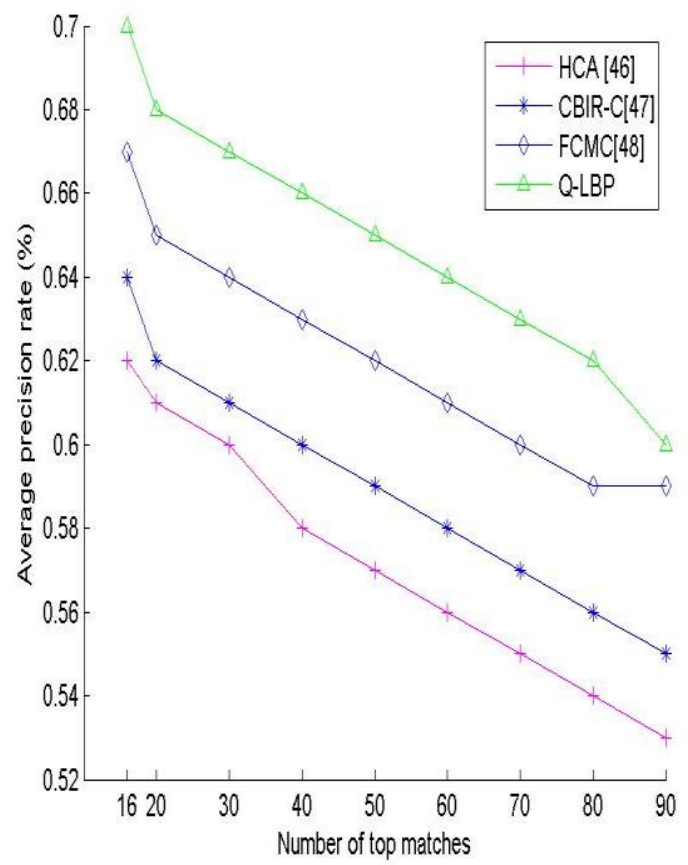

Fig. 9: Average Performance curve (precision) using HCA, CBIR-C, FCMC and proposed integrated Q-LBP method.

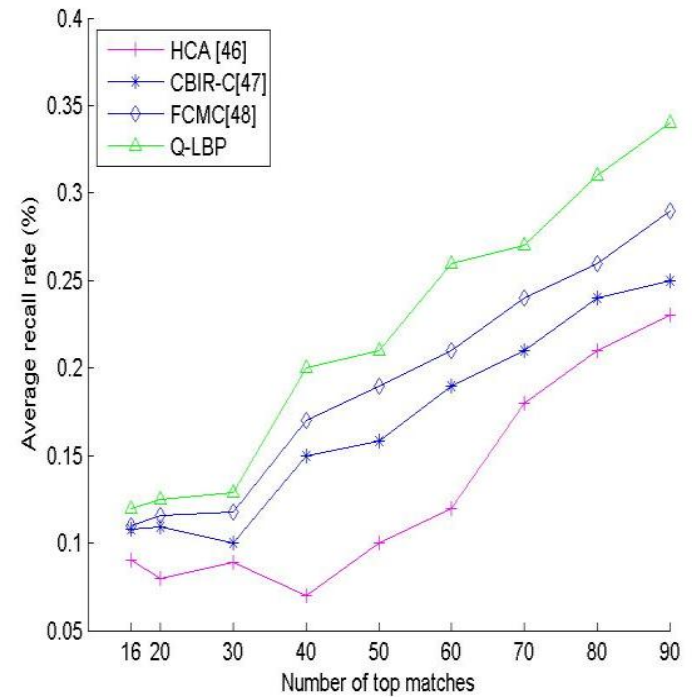

Fig. 10: Average Performance curve (recall) using HCA, CBIR-C, FCMC and proposed integrated Q-LBP method.

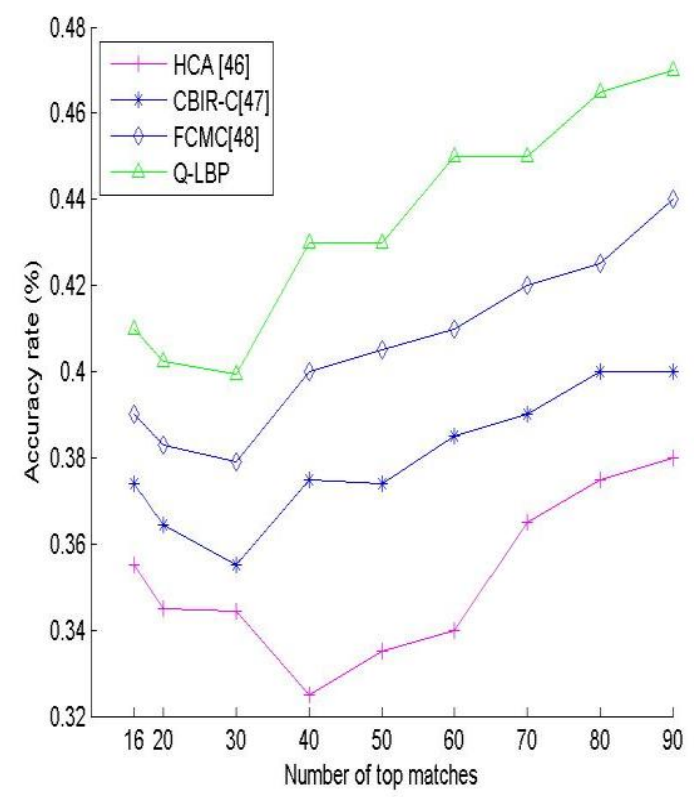

Fig. 11: Average Performance curve (accuracy) using HCA, CBIR-C, FCMC and proposed integrated Q-LBP method.

\section{CONCLUSIONS}

The proposed integrated Q-LBP CBIR model integrated the features from texture, shape and color. The statistical approaches gained lot of significance in image retrieval and LBP based methods raises high dimensionality and complexity issues, in deriving statistical features. The present paper addresses these two issues by quantizing the LBP code, to reduce dimensionality and by deriving GLCM features on quantized LBP. The proposed model is robust. The proposed method is carried out with varying distances and number of retrieved images. The proposed method shown high results of retrieval for $\mathrm{D}=4$ and it outperforms the other existing methods. 


\section{REFERENCES}

[1] Y. Rui and T. S. Huang, Image retrieval: Current techniques, promising directions and open issues, J.. Vis. Commun. Image Represent., 10 (1999) 39-62.

[2] A.W.M. Smeulders, M. Worring, S. Santini, A. Gupta, and R. Jain, Content-based image retrieval at the end of the early years, IEEE Trans. Pattern Anal. Mach. Intell., 22 (12) 1349-1380, 2000

[3] Alnihoud J., "Content-Based Image Retrieval System Based on Self Organizing Map, Fuzzy Color Histogram and Subtractive Fuzzy Clustering," the International Arab Journal ofInformation Technology, vol. 9, no. 5, pp. 452458, 2012.

[4] Jain A. and Farrokhnia F., "Unsupervised Texture Segmentation using Gabor Filters," InProceedings of IEEE International Conferenceon Systems Man and Cybernetic, Los Angeles, USA, pp. 14-19, 1990.

[5] M. Kokare, B. N. Chatterji, P. K. Biswas, A survey on current content based image retrieval methods, IETE J. Res., 48 (3\&4) 261-271, 2002.

[6] Ying Liu, Dengsheng Zhang, Guojun Lu, Wei-Ying Ma, Asurvey of content-based image retrieval with high-level semantics, Elsevier J. Pattern Recognition, 40, 262-282, 2007.

[7] M. J. Swain and D. H. Ballar, Indexing via color histograms, Proc. 3rd Int. Conf. Computer Vision, Rochester Univ., NY, (1991) 11-32.

[8] M. Stricker and M. Oreng, Similarity of color images, Proc. SPIE, Storage and Retrieval for Image and Video Databases, (1995) 381-392.

[9] G. Pass, R. Zabih, and J. Miller, Comparing images using color coherence vectors, Proc. 4th ACM Multimedia Conf., Boston, Massachusetts, US, (1997) 65-73.

[10] J. Huang, S. R. Kumar, and M. Mitra, Combining supervised learning with color correlograms for contentbased image retrieval, Proc. 5th ACM Multimedia Conf., (1997) 325-334.

[11] Z. M. Lu and H. Burkhardt, Colour image retrieval based on DCT domain vector quantization index histograms, J. Electron. Lett., 41 (17) (2005) 29-30.

[12] Chen Y., Wang J., and Krovetz R., "Content Based Image Retrieval by Clustering," in Proceedings of 5th International ACM Workshop on Multimedia Information Retrieval, pp. 193-200, 2003.

[13] Monika J. and Singh S., "An Experimental Study on Content Based Image Retrieval Based on Number of Clusters using Hierarchical Clustering Algorithm," International Journal of SignalProcessing, Image Processing and PatternRecognition, vol. 7, no. 4, pp. 105$114,2014$.

[14] Pavani P. and Prabha S., "Content Based Image Retrieval using Machine Learning Approach," in Proceedings of the International Conference on Frontiers of Intelligent Computing: Theory and Applications, vol. 247, pp. 173179, 2013.

[15] Y Venkateswarlu, B Sujatha, V Vijaya Kumar, Image classification based on center symmetric fuzzy texture unit matrix, International journal of scientific \& engineering research, Vol.13, No.11, pp.5-11, Oct-2012, ISSN 2229-5518.

[16] V.Vijaya Kumar, B. Eswar Reddy, U.S.N. Raju, K. Chandra Sekharan,An innovative technique of texture classification and comparison based on long linear patterns, Journal of computer science, Science publications, Vol.3, No.8, pp.633-638, Aug-2007, ISSN $1552-6607$.

[17] V Vijaya Kumar, U S N Raju, K Chandra Sekaran, V V Krishna, Employing long linear patterns for texture classification relying on wavelets, ICGST-Graphics, vision and image processing (ICGST-GVIP), Vol.8,No.5, pp. 13-21, Jan-2009, ISSN: 1687-398X

[18] V. Vijaya Kumar, K. Srinivasa Reddy, V. Venkata Krishna, Face Recognition Using Prominent LBP Model; International Journal of Applied Engineering Research Vol. 10, Issue 2, pp. 4373-4384, 2015, ISSN: 0973-4562

[19] M. Chandra Mohan,V. VijayaKumarU.S.N. Raju,New face recognition method based on texture features using linear wavelet transforms, International journal of computer science and network security (IJCSNS), Vol.9 No.12, pp.223-230, Dec-2009, ISSN: 1738-7906.

[20] P. Chandra SekharReddy,B. EswaraReddy,V. Vijaya Kumar, Fuzzy based image dimensionality reduction using shape primitives for efficient face recognition, ICTACT- Journal on image and video processing, Vol 04, NO. 02, pp. 695-701, Nov-2013, ISSN: 0976-9102.

[21] V Vijaya Kumar, GortiSatyanarayaMurty, PS V V S R Kumar, Classification of facial expressions based on transitions derived from third order neighborhood LBP, Global journal of computer science and technology graphics \& vision (GJCST), Vol.14, N0.1, pp. 1-12, Jan2014, ISSN: 0975-4350.

[22] G S Murty ,J SasiKiran , V.Vijaya Kumar, Facial expression recognition based on features derived from the distinct LBP and GLCM, International journal of image, graphics and signal processing (IJIGSP), , Vol.2, No.1, pp. $68-77,2014$, ISSN: $2074-9082$.

[23] V.VijayaKumar,SakaKezia, I.SantiPrabha,A new texture segmentation approach for medical images, International journal of scientific \& engineering research, Vol. 4, No.1, pp.1-5, January-2013, ISSN: 2229-5518.

[24] V.Vijaya Kumar, U.S.N. Raju, M. Radhika Mani, A. NarasimhaRao,Wavelet based texture segmentation methods based on combinatorial of morphological and statistical operations, International journal of computer science and network security (IJCSNS), Vol.8, No.8, pp.176-181, Aug-2008, ISSN : 1738-7906.

[25] SakaKezia, I.SantiPrabha, V.VijayaKumar,A color-texture based segmentation method to extract object from background, International journal image, and graphics and signal processing, Vol. 5, No. 3, pp.19-25, March-2013, ISSN: 2074- 9082.

[26] NG rao,DVVKDPSVS Rao, Novel Approaches of evaluating Texture Based Similarity Features for Efficient Medical Image Retrieval System, International Journal Of Computer Applications, ISSN-0975-8887 20(7),20-26

[27] A Obulesu,JSKiran, VV Kumar, Facial image retrieval based on local and regional features, IEEE- 2015 International Conference on Applied and Theoretical 
Computing and Communication Technology (iCATccT), 29-31 Oct. 2015, Pp:841 - 846

[28] V.Vijaya Kumar, A. Srinivasa Rao, YK Sundara Krishna, Dual Transition Uniform LBP Matrix for Efficient Image Retrieval, I.J. Image, Graphics and Signal Processing, 2015, 8, 50-57.

[29] V. Vijaya Kumar, N. Gnaneswara Rao, A.L.Narsimha Rao, And V.Venkata Krishna4,IHBM: Integrated histogram bin matching for similarity measures of color image retrieval, International journal of signal processing, image processing and pattern recognition, Vol. 2, No.3, pp. 109-120, Sep-2009, ISSN: 2005-4254.

[30] He X., Ma W., and Zhang H., "Learning an Image Manifold for Retrieval," in Proceedings ofACM International Conference on Multimedia,New York, USA, pp. 17-23, 2004.

[31] Chen Y., Wang J., and Krovetz R., "An Unsupervised Learning Approach to Content-Based Image Retrieval," in proceeding of 7th International Symposium on SignalProcessing and its Applications, pp. 197-200, 2003.

[32] Kim D. and Chung C., "Qcluster: Relevance Feedback using Adaptive Clustering for Content Based Image Retrieval," in Proceedings of the ACM SIGMOD'03, San Diego, USA, pp. 599-610, 2003.

[33] J. R. Smith and S. F. Chang, Automated binary texture feature sets for image retrieval, Proc. IEEE Int. Conf. Acoustics, Speech and Signal Processing, Columbia Univ., New York, (1996) 2239-2242.

[34] H. A. Moghaddam, T. T. Khajoie, A. H Rouhi and M. Saadatmand T., Wavelet Correlogram: A new approach for image indexing and retrieval, Elsevier J. Pattern Recognition, 38 (2005) 2506-2518.

[35] H. A. Moghaddam and M. Saadatmand T., Gabor wavelet Correlogram Algorithm for Image Indexing and Retrieval, 18th Int. Conf. Pattern Recognition, K.N. Toosi Univ. of Technol., Tehran, Iran, (2006) 925-928.

[36] A.Ahmadian, A. Mostafa, An Efficient Texture Classification Algorithm using Gabor wavelet, 25th Annual international conf. of the IEEE EMBS, Cancun, Mexico, (2003) 930-933.

[37] H. A. Moghaddam, T. T. Khajoie and A. H. Rouhi, A New Algorithm for Image Indexing and Retrieval Using Wavelet Correlogram, Int. Conf. Image Processing, K.N. Toosi Univ. of Technol., Tehran, Iran, 2 (2003) 497-500.

[38] M. Saadatmand T. and H. A. Moghaddam, Enhanced Wavelet Correlogram Methods for Image Indexing and Retrieval, IEEE Int. Conf. Image Processing, K.N. Toosi Univ. of Technol., Tehran, Iran, (2005) 541-544.

[39] M. Saadatmand T. and H. A. Moghaddam, A Novel Evolutionary Approach for Optimizing Content Based Image Retrieval, IEEE Trans. Systems, Man, and Cybernetics, 37 (1) (2007) 139-153.

[40] L. Birgale, M. Kokare, D. Doye, Color and Texture Features for Content Based Image Retrieval, International Conf. Computer Grafics, Image and Visualisation, Washington, DC, USA, (2006) 146 - 149.

[41] M. Subrahmanyam, A. B. Gonde and R. P. Maheshwari, Color and Texture Features for Image Indexing and
Retrieval, IEEE Int. Advance Computing Conf., Patial, India, (2009) 1411-1416.

[42] SubrahmanyamMurala, R. P. Maheshwari, R. Balasubramanian, A Correlogram Algorithm for Image Indexing and Retrieval Using Wavelet and Rotated Wavelet Filters, Int. J. Signal and Imaging Systems Engineering.

[43] R.M. Haralick, K. Shangmugam, I. Dinstein, Textural feature for image classification, IEEE Trans. Syst. Man Cybern. SMC-3 (6) (1973) 610-621.

[44] Coggins J.M. and Jain A.K., "A spatial filtering approach to texture analysis," Pattern Recogninon Letters, no.3, pp.195-203, 1985 .

[45] http://wang.ist.psu.edu/.

[46] K. Jarrah, S. Krishnan, L. Guan , "Automatic contentbased image retrieval using hierarchical clustering algorithms," in Proc. International Joint Conference on Neural Networks (IJCNN '06), Oct. 2006, Vancouver, BC pp. 3532 - 3537.

[47] Ms. Urvashi Chavan1, Prof. N. M. Shahane2, Content Based Image Retrieval Using Clustering, International Journal of Application or Innovation in Engineering \& Management (IJAIEM), Volume 3, Issue 10, October 2014

[48] L. Pengyu, J. Kebin, Z. Peizhen, "An effective method of image retrieval based on modified Fuzzy C-Means clustering scheme", Proceeding ICSP2006.

\section{AUTHOR PROFILE}

P. Latha Reddy received the B.Tech. degree in Information Technology from, Indur Institute Of Engineering And Technology, Siddipet in 2014 and is currently persuing Master's Degree in Computer Science and Engineering from Anurag Groups Of Institutions(CVSR), Hyderabad.

Dr. V. Vijaya Kumar is working as Dean in Dept. of CSE \& IT, chairman Board of studies for CSE and IT and Director Centre for Advanced Computational Research (CACR) at Anurag Group of Institutions, (AGOI) (Autonomous), Hyderabad. He received integrated M.S.Engg, in CSE from USSR in 1989. He received his Ph.D. degree in Computer Science from Jawaharlal Nehru Technological University (JNTU), Hyderabad, India in 1998 and guided 28 research scholars for $\mathrm{Ph} . \mathrm{D}$. He acted as principle investigator for various $R \& D$ projects. He has served JNT University for 13 years as Assistant Professor, Associate Professor and Professor. He has received best researcher and best teacher award from JNT University, Kakinada, India. His research interests include big data, image analytics, image retrieval, image processing, pattern recognition, digital water marking and cloud computing. At present he is also acting as BoS member for JNTUH and various other institutions. He is the life member of CSI, ISCA,

ISTE, IE (I), IETE, ACCS, CRSI, IRS and REDCROSS. He published more than 100 research publications till now in various national, international journals and conferences. He has delivered key note addresses at various international conferences.

A.Obulesu is working as Asst.Prof at Anurag Group of Institutions (AGI) (Autonomous), Hyderabad and Graduated in B.Tech from Nagarjuna Institute of Technology, Vijayawada which is affiliated to JNTU Hyderabad in 2003. He received 
Masters Degree in M.Tech. from Rajeev Gandhi Memorial College of Engineering and Technology, Nandyal which is affiliated to JNT University, Hyderabad, in 2006. At present he is pursuing Ph.D. from JNTU Kakinada in Image Processing under the guidence of Dr.Vakulabharanam Vijaya Kumar,

Director - Centre for Advanced Computational Research
(CACR) of Anurag Group of Institutions (Autonomous), Hyderabad and an active member in CACR. He has published 15 research papers in various National, International journals and conferences proceedings. 